\title{
COMMUNICATIONS
}

\section{Electron emission from deep level defects EL2 and EL6 in semi- insulating GaAs observed by positron drift velocity transient measurements}

\author{
J. M. Tsia, C. C. Ling, ${ }^{\text {a) }}$ C. D. Beling, and S. Fung \\ Department of Physics, The University of Hong Kong, Pokfulam Road, Hong Kong, China
}

(Received 26 April 2002; accepted for publication 3 July 2002)

\begin{abstract}
A $\pm 100 \mathrm{~V}$ square wave applied to a Au/semi-insulating SI-GaAs interface was used to bring about electron emission from and capture into deep level defects in the region adjacent to the interface. The electric field transient resulting from deep level emission was studied by monitoring the positron drift velocity in the region. A deep level transient spectrum was obtained by computing the trap emission rate as a function of temperature and two peaks corresponding to EL2 ( $E_{a}=0.81$ $\pm 0.15 \mathrm{eV})$ and EL6 $\left(E_{a}=0.30 \pm 0.12 \mathrm{eV}\right)$ have been identified. (C) 2002 American Institute of Physics. [DOI: 10.1063/1.1503162]
\end{abstract}

Various spectroscopic techniques have been developed under the general heading of positron annihilation spectroscopy (PAS) for the purpose of studying open volume defects in solids. ${ }^{1-3}$ One of the less explored methods of employing positrons to study defects in semiconductors was suggested in 1994 as a result of measuring the apparent positron mobility at Au/semi-insulating SI-GaAs contact. ${ }^{4}$ The presence of the EL2 deep donor was revealed in these measurements through an anomalous peak in the apparent positron mobility around $300 \mathrm{~K}$ that depended on the frequency of the applied ac field. The anomalous peak resulted from a temperature dependent build up of EL2 ${ }^{+}$space charge that caused the thermalized positrons to experience a strong electric field back towards the positron injecting contact. Arrhenius analysis of the temperature movement of the mobility peak gave an energy of the emitting trap $E_{C}-0.8 \mathrm{eV}$ which identified the trap as EL2 ${ }^{+} .5$

In this study, a symmetric $\mathrm{Au} / \mathrm{SI}-\mathrm{GaAs} / \mathrm{Au}$ structure with Schottky-like contacts was pulsed with a square wave, which permitted deep trap emission and capture processes to occur. As in previous studies of this nature, ${ }^{4}$ the dynamics of the electron capture and emission from the deep traps determined the electric field in the space charge region, which in turn was revealed by measurement of the Doppler shift on the annihilation gamma photons from the positron drifting in the electric field. By monitoring the positron drift velocity as a function of time at different temperatures, not only could the dominant GaAs defect EL2 be detected, but the transients taken in the course of the present work also revealed the deep trap EL6.

The Au/SI-GaAs/Au structure was fabricated as in previous studies. ${ }^{4} \mathrm{Au}$ was thermally evaporated in a vacuum of $10^{-6}$ mbarr onto both sides of $1 \times 1 \mathrm{~cm}^{2}$ sample cut from a (100) oriented LEC grown SI-GaAs wafer (resistivity=

a)Electronic mail: ccling@hku.hk
$10^{8} \Omega \mathrm{cm}$, thickness $=0.5 \mathrm{~mm},[\mathrm{EL} 2]=1.5 \times 10^{16} \mathrm{~cm}^{-3}$ and $[C]=10^{15} \mathrm{~cm}^{-3}$ ) purchased from MCP Wafer Technology Ltd. The contacts were subject to no annealing. The positron source was $20 \mu \mathrm{Ci}$ of ${ }^{22} \mathrm{NaCl}$ encapsulated in a Kapton foil. This radioactive source foil was sandwiched between the sample piece and a piece of $0.5 \mathrm{~mm}$ thick lead, the function of which was to backscatter a significant fraction $(\sim 50 \%)$ of positrons emitted from the source into the GaAs. The whole sample assembly was loaded into a $10 \mathrm{~K}$ closed cycle $\mathrm{He}$ fridge. The gamma photons were detected by a 30\% HPGe gamma detector. The method of measuring the positron drift velocity was originated from Mills et al. ${ }^{6}$ and has also been implemented in our previous study where a more detailed description may be found. ${ }^{4}$ In brief, annihilation photons originating from positrons drifting in an electric field are Doppler shifted with an energy of $\Delta E / E=v_{+} / 2 c$, where $v_{+}$is the positron drift velocity longitudinal to the detector. In the present study, the fractional change $\Delta \mathrm{E} / \mathrm{E}$ is as small as $10^{-5}$ while the electric field and the positron mobility are of the orders $\sim 10 \mathrm{kV} \mathrm{cm}^{-1}$ (Refs. 7-11) and $\sim 100$ $\mathrm{cm}^{2} \mathrm{~V}^{-1} \mathrm{~s}^{-1}$ (Table III in Ref. 8). The amplified signal from the $\mathrm{Ge}$ detector is passed to two signal channel analyzers (namely $\mathrm{SCA}_{1}$ and $\mathrm{SCA}_{2}$ ). The energy windows of $\mathrm{SCA}_{1}$ and $\mathrm{SCA}_{2}$ were set at positions such that they equally bisected the whole energy photopeak as there was no positron drift. If $N_{1}$ and $N_{2}$ designate the counts from the low and high energy windows, respectively, then the Doppler shift of the gamma photon is revealed by the small changes in $N_{1}$ and $N_{2}$. These two singles rates $\left(\sim 3 \times 10^{3} \mathrm{~s}^{-1}\right)$ were recorded by a sweeping ORTEC multichannel scaler MCS synchronized to a Lakeshore 330 function generator giving the $\pm 100 \mathrm{~V}$ square wave bias to the sample. The positron drift velocity is given by Ref. 6: $\mathrm{v}_{+}=\frac{1}{2} \alpha\left[\left(\left\langle N_{1}\right\rangle-N_{1}\right) /\left\langle N_{1}\right\rangle\right.$ $\left.-\left(\left\langle N_{2}\right\rangle-N_{2}\right) /\left\langle N_{2}\right\rangle\right]$, where $\left\langle N_{i}\right\rangle$ is the average count and $\alpha$ is the calibration factor. A total number of $10^{9}$ events were collected (in about four days) in both capture and emission 


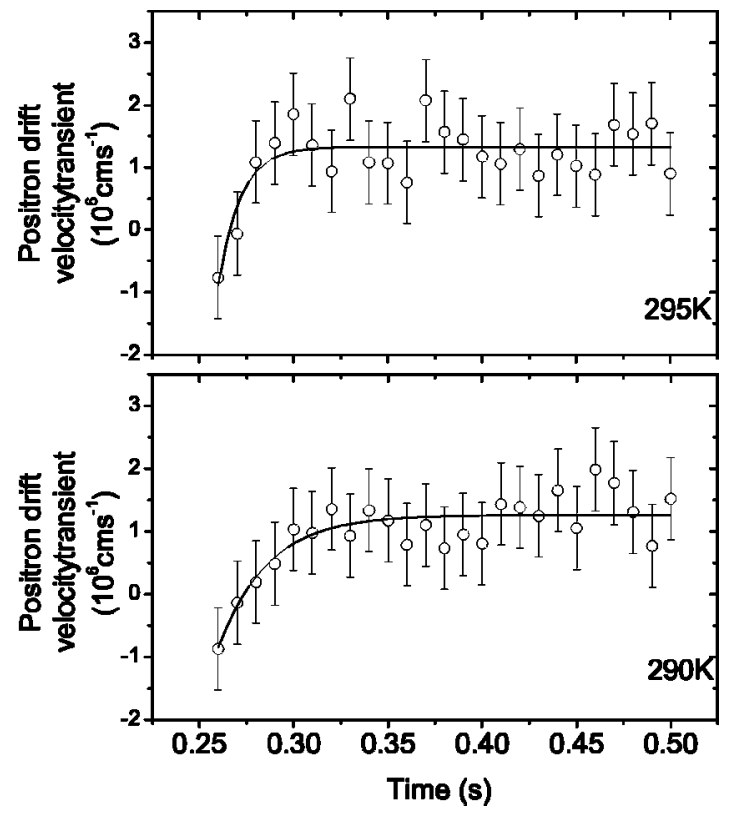

FIG. 1. Positron drift velocity transient in the emission half period at $\mathrm{T}=290 \mathrm{~K}$ and $\mathrm{T}=295 \mathrm{~K}$. The solid lines are the fitted curves using the relation of $1-\exp \left(-e_{n} t\right)$. It clearly shows the faster emission rate with higher temperature. The zero position of the positron drift velocity axis shown in the figure is shifted due to the system stabilization that equalizing $\mathrm{N}_{1}$ and $\mathrm{N}_{2}$.

cycles. The sweep time was $500 \mathrm{~ms}$. The whole energy spectrometer was stabilized with an attenuating stabilizer that changed the effective gain of the system amplification so as to maintain an equal $\mathrm{N}_{1}$ and $\mathrm{N}_{2}$ with a relaxation time $(\sim 100$ s) much longer than the sweep period.

A forward bias applied to the positron injection $\mathrm{Au} / \mathrm{GaAs}$ contact in the first $250 \mathrm{~ms}$ (capture) half cycle causes the electron quasi-Fermi energy to increase in height and the ionized EL2 ${ }^{+}$centers in the GaAs to neutralize. This neutralization process causes the electric field and observed positron drift velocity to decay to close to zero in times $\sim 100 \mathrm{~ms}$. In the second $250 \mathrm{~ms}$ (emission) half cycle the $\mathrm{Au} / \mathrm{GaAs}$ contact closest to the positron source, being under effective reverse bias conditions, becomes depleted of electrons and the EL2 defect ionizes to EL2 ${ }^{+}$. The increasing space charge and resulting electric field cause the dominant "blueshifted" positron velocity transient. Positron drift velocity transients taken at 290 and $295 \mathrm{~K}$ in the emission half period (i.e., $t=0.25-0.50 \mathrm{~s})$ are shown in Fig. 1 .

From visual inspection of Fig. 1, the rate of increase of positron velocity with time in the emission half cycle increases while temperature increases from 290 to $295 \mathrm{~K}$ and this is also a general trend for all the transient data taken at temperatures ranging between 280 and $295 \mathrm{~K}$. This observation may be understood from the Arrhenius dependence of the trap emission rate: ${ }^{12} \quad e_{n}=\sigma_{n} v_{n_{0}} N_{C_{0}}\left(T / T_{0}\right)^{2} \exp$ $\left(-E_{\text {act }} / k T\right)$, where $v_{n_{0}}$ and $N_{C_{0}}$ are, respectively, the thermal carrier velocity and the conduction state density at a temperature of $T_{0}$. The emission from and the capture into deep level defects can be described by the rate equation: $d N_{+} / d t=-e_{n}\left(N_{0}-N_{+}\right)+c_{n} N_{+}$, where $c_{n}$ is the capture rate of the deep level, $N_{0}$ and $N_{+}$are the total and ionized

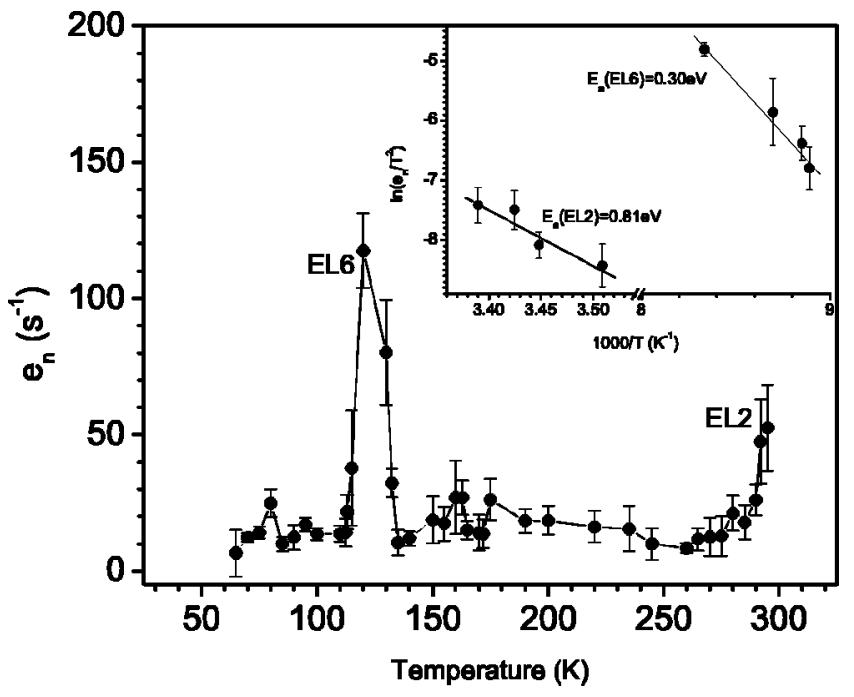

FIG. 2. Deep level transient spectrum obtained by plotting the emission rate as a function of temperature. Two peaks, namely the EL2 and EL6, are clearly observed at $\mathrm{T} \sim 120 \mathrm{~K}$ and $\mathrm{T} \sim 300 \mathrm{~K}$. The Arrhenius plots of the two peaks are shown in the small figure at the corner and the activation energies were determined to be $0.30 \pm 0.12 \mathrm{eV}$ (EL6) and $0.81 \pm 0.15 \mathrm{eV}$ (EL2).

deep level concentrations respectively. Assuming that in the emission half cycle $c_{n} \sim 0$ the rate equation integrates to give $N_{+}(t)=N_{0}\left[1-\exp \left(-e_{n} t\right)\right]$. It has been known for some time that, because SI-GaAs lies in the regime of being a relaxation semiconductor, ${ }^{13,14}$ one cannot apply the standard depletion model approximation to determine the electric field structure at a metal contact. ${ }^{7-11}$ Indeed the $\mathrm{Au}-\mathrm{SI}-\mathrm{GaAs}$ contact under reverse bias shows a much larger than that expected depletion region and an electric field that saturates at a critical value of $\xi_{\text {sat }} \sim 10 \mathrm{kV} \mathrm{cm}{ }^{-1}$ for reasons that are still not fully substantiated. ${ }^{7-11}$ The electric field thus remains fairly constant over the sample extending over about a hundred microns and then drops-off rather sharply over an $\sim 50 \mu \mathrm{m}$ thick space charge region to the low bulk electric field value. These observations have been attributed to the enhanced EL2 ${ }^{+}$electron capture cross section for more energetic electrons. ${ }^{9}$ As far as we know there have been no transient studies made of how the EL2 traps behave at the metal/SI-GaAs structure shortly after the application of a reverse bias. The depletion of electrons leads one to suppose that the behavior $N_{+} \sim 1-\exp \left(-e_{n} t\right)$ will occur, but then at some point the buildup in electric field must self quench causing the strong field and depletion zone to propagate deeper into the bulk. For the moment it is assumed that to a reasonable approximation, the main portion of space charge accumulates at a deeper distance than the mean positron implantation depth. This implies that at time well before the electric field reaches the saturated value $\xi_{\text {sat }} 10 \mathrm{kV} \mathrm{cm}^{-1}$ such that the high field enhanced capture occurs, the velocity transient becomes: $v_{+}(t) \propto\left[1-\exp \left(-e_{n} t\right)\right]$. Such exponential fits are seen to give good fits to the experimental data in Fig. 1 and as shown in the fitted curves, the deep trap EL2 emits with a faster rate at higher temperature, which is of the expectation of the temperature dependence of $e_{n}$.

Emission rates at different temperatures were obtained by fitting the emission transient data according to $v_{+}(t)$ 
$\propto\left[1-\exp \left(-e_{n} t\right)\right]$ at different temperatures from 65 to $295 \mathrm{~K}$. The results are shown in Fig. 2, where it may be seen that the emission rate drops with decreasing temperature at temperature around $295 \mathrm{~K}$ and it is suggestively related to the activation of the deep level defect EL2. The activation energy $\mathrm{E}_{\mathrm{act}}$ can thus be deduced by the Arrhenius plot $\ln \left(e_{n} / \mathrm{T}^{2}\right)$ against 1/T. An Arrhenius plot made in the temperature region 285 $\mathrm{K}-295 \mathrm{~K}$ is shown in Fig. 2 and give the deep level activation energy as $0.81 \pm 0.15 \mathrm{eV}$, which is close to the generally accepted value of the second thermal ionization energy of EL2. ${ }^{15}$

It is also interesting to show that in Fig. 2, other than EL2 signal at $T \sim 295 \mathrm{~K}$, another peak at about $120 \mathrm{~K}$ can also been seen. This peak cannot be due to the EL2 deep level, where the EL2 emission can only be slowed down at this low temperature. This implies that the $120 \mathrm{~K}$ peak is related to another deep level defect which is shallower than EL2. The emission rate of this defect, which is too fast at $\mathrm{T} \sim 290 \mathrm{~K}$, is slowed down at the peaking temperature $\mathrm{T}_{\text {peak }}$ $\sim 120 \mathrm{~K}$ and gives response in the transient time window equal to $250 \mathrm{~ms}$. In other words, the $e_{n}$ is close to zero as $T \ll T_{\text {peak }}$. $e_{n}$ becomes large so as to allow transient to fill the time window at $T \sim T_{\text {peak }}$. As $T \gg T_{\text {peak }}, e_{n}$ is so large that it is faster than the time binning, transient cannot be seen and the measured $e_{n}$ becomes zero. The Arrhenius plot of this peak is plotted and shown in the box of Fig. 2 and the activation energy is determined to be $0.30 \pm 0.12 \mathrm{eV}$, which is in good agreement with the normal range of values for the EL6 activation energy $\left(E_{a}=0.32 \mathrm{eV}\right) .^{15}$

It should be pointed out that the positron velocity transient technique described here is different from another versatile PAS transient technique discussed in Ref. 5. The latter method depends on looking for transients in the conventional positron lifetime and Doppler broadening measurements after the application of a pulsed excitation (e.g., photoexcitation of the defect) and the observation of these transients gives indication of the presence of excitable vacancy defects. The positron velocity transient technique used in this work relies on the fact that the positron experiences an increasing electric field arising from the buildup of space charge. Although the present technique gives little additional information over the conventional DLTS, it does have an advantage when the trap density exceeds the donor concentration in which case the latter would fail. Moreover this new tech- nique can now be added to the general family of PAS as an additional probe for nonopen volume deep level defects and, in doing so, extending the PAS defect detection limit to beyond $10^{15} \mathrm{~cm}^{-3}$.

In the present study, we have demonstrated how positron drift velocity transient can be used to study the deep level defects in semi-insulating GaAs. In this technique, the electric field transient arising from the emission and capture processes, as the Schottky contact is under a square wave bias, is monitored by the positron drift velocity which is measured by observing the Doppler shift of the annihilation photon energy. The emission rate as a function of temperature can be found and the activation energy of the deep level defect is deduced from the Arrhenius plot. Both EL2 and EL6 have been detected in the SI-GaAs material and their activation energies have been calculated to be $0.81 \pm 0.15$ and 0.30 $\pm 0.12 \mathrm{eV}$, respectively.

The work described in this communication is supported by Grants from the Research Grant Council of the Hong Kong Special Administrative Region, China (under Project Nos. HKU7090/97P, HKU1/00C, and HKU7137/99P) and the HKU CRCG.

${ }^{1}$ P. J. Schultz and K. G. Lynn, Rev. Mod. Phys. 60, 701 (1988).

${ }^{2}$ M. J. Puska and R. M. Nieminen, Rev. Mod. Phys. 66, 841 (1994).

${ }^{3}$ R. S. Krause-Rehberg and H. S. Leipner, Positron Annihilation in Semiconductors, Defect Studies, Springer Series in Solid-State Sciences 127 (Springer, Berlin, 1999).

${ }^{4}$ H. L. Au, C. C. Ling, P. K. Panda, T. C. Lee, C. D. Beling, and S. Fung, Phys. Rev. Lett. 73, 2732 (1994).

${ }^{5}$ C. D. Beling, S. Fung, H. L. Au, C. C. Ling, C. V. Reddy, A. H. Deng, and B. K. Panda, Appl. Surf. Sci. 116, 121 (1997).

${ }^{6}$ A. P. Mills, Jr., E. M. Gulliksen, L. Pfeiffer, and W. S. Rockward, Phys. Rev. B 33, 7799 (1986).

${ }^{7}$ C. C. Ling, Y. F. Shek, A. P. Huang, S. Fung, and C. D. Beling, Phys. Rev. B 59, 5751 (1999).

${ }^{8}$ Y. F. Hu, C. C. Ling, C. D. Beling, and S. Fung, J. Appl. Phys. 82, 3891 (1997).

${ }^{9}$ D. S. McGregor, R. A. Rojeski, G. F. Knoll, F. L. Terry, Jr., J. East, and Y. Eisen, J. Appl. Phys. 75, 7910 (1994).

${ }^{10}$ K. Berwick, M. R. Brozel, C. M. Buttar, M. Cowperthwaite, P. Sellin, and Y. Hou, Mater. Sci. Eng., B 28, 485 (1994).

${ }^{11}$ A. Castaldini, A. Cavallini, L. Polenta, C. Canali, C. del Papa, and F. Nava, Phys. Rev. B 56, 9201 (1997).

${ }^{12}$ M. Lannoo and J. Bourgoin, Point Defects in Semiconductor II (Springer, Berlin, 1983).

${ }^{13}$ N. M. Haegel, Appl. Phys. A: Solids Surf. 53, 1 (1991).

${ }^{14}$ J. Santana and B. K. Jones, J. Appl. Phys. 83, 7699 (1998).

${ }^{15}$ G. M. Martin, A. Mitonneau, and A. Mircea, Electron. Lett. 13, 191 (1977) 